\title{
Research on the Reference and Acceptance of Yangshen to Guopo's Shanhai Jing Note
}

\author{
Shuyan $\mathrm{Yi}^{1, \mathrm{a}}$, Hao Wang ${ }^{1, \mathrm{~b}}$, Yao $\mathrm{Xu}^{2, \mathrm{c}}$ \\ ${ }^{1}$ College of Humanities \&Sciences of Northeast Normal University, Changchun 130117, China; \\ ${ }^{2}$ School of Changchun University of Technology, Changchun 130012, China. \\ ayishuyan2013@126.com, b348637511@qq.com, cxuyao@ccut.edu.cn
}

Keywords: Shanhai Jing Note; Guopu; Yangshen; Reference; Acceptance.

\begin{abstract}
Guopu is a famous scholar in the Jin Dynasty; his Shanhai Jing Note is the earliest and the most authoritative note of Shanhai Jing, which has the effects of Yang Shen's note on the following aspects. first, Nature identification: In his learned perspective, Guo Pujian believed that what the Shanhai Jing signed is true; Second, In terms of the content of comments, they both paied attention to the textual analysis and textual research object; Third, In terms of the annotation method, they both use the information obtained by themselves to prove Shanhai Jing's authenticity; Fourth, they both have the spirit of seeking truth and pragmatism. Through this comparative study, which not only can deepen the research on Shanhai Jing's text, can also deepen the understanding of Guo Pu, Yang Shen and his note book.
\end{abstract}

\section{Introduction}

As the first famous note person of Shanhai Jing, The Eastern Jin Dynasty scholar Guopu has great contribution on Shanhai Jing's spread and research, and whose Shanhai Jing note has an important research value. In the course of spreading, his Shanhai Jing note has become an integral whole with the original text. ${ }^{[1][2]}$ Most of the scholars are based on his note book to make new notes. Under the influence of the special age atmosphere, Shanhai Jing had become the focus of attention of scholars in the Ming Dynasty. Among which, Yangshen's Additional Note of Shanhai Jing is similar with Guopu's Shanhai Jing note. This paper would discuss on the following aspects of the impact of GuoPu's Shanhai Jing notes on Yangshen. ${ }^{[3]}$

\section{The reference and acceptance of Yangshen to Guopo's Shanhai Jing Note}

\subsection{Under the horizon of knowledge, believed the truth of Shanhai Jing.} Although in the Han Dynasty experienced a "study Confucian literature" period, but in a long time, because of the content of the unreal and strange, Shanhai Jing was not to be valued by traditional scholars. Because of his special experience "Yi Jiangnan", wide range of knowledge, Guo Pu's vision is higher than the general scholars. This makes Guo Pu the brave to jump out of the traditional nest to have some creative literary creations. ${ }^{[4]}$ On the begin of he noted Shanhai Jing, Guo Pu believes that Shanhai Jing is an authentic Museum Book, and identified the author of Shanhai Jing as Xia Yu. Guo $\mathrm{Pu}$ proposed the idealism argument as "there is nothing different, it is only because of the viewer's different", he not only use Zhuangzi's "the knowledge of people, if they don't know" as a theoretical support, also through listed the Han Dynasty, Jin various facts, and cited the ancient literature as the evidence to prove his point. ${ }^{[5]}$

It is to see the full nature of Guo Pu's argument, the later orthodox historians and scholars more considered Shanhai Jing as an history - geography book, such as the "Sui Shu- Ji Zhi” , the "Old Tang - Ji Zhi” , the "Xintangshu - Yiwenzhi”, the " Chongwen head ", etc. This thought has been the mainstream until the Southern Song Dynasty.

Yang Shen has a profound knowledge and rich traveling experience, and his has the same point in the understanding of Shanhai Jing with Guo Pu. In the time of being banished to Yunnan for 38 years, 
in addition to his accomplishments in poetry and music, Yang Shen also did some in-depth study on the history books and the literature in all areas of all classes of authors.

Like Guo $\mathrm{Pu}$, the inauspicious official career's make Yang Shen's focus on learning, trying to achieve liberation and value seeking spirit from the book of Zhuangzi. This liberal attitude makes Yang Shen Give a high degree of evaluation to Shanhai Jing, he said: "the six channels are as grain to people, nobody can live with grain. Some books out of the six channels are the feast of fat things to the scholars, such as Wenxuan and Shanhai Jing.” The point of his put Shanhai Jing the position forward of the six channels showed that he is full affirmation of Shanhai Jing. In the annotation process, Yang Shen paied great attention to the realistic part of the content, and able to using the information he has received in his notes, in order to demonstrate its absurd unreal "Haijing" "Huangjing" of the authenticity of the content. ${ }^{[6]}$

\subsection{Pay attention to exegesis and textual research object.}

As the Jin Dynasty famous exegesis, Guo Pu not only focused on the text, sound, meaning the exegesis, but also attached great importance to the mountains and other textual researches. "Exegesis" and "textual research" are the two main content of Guo Pu's Shanhai Jing note. Which not only give direction for future generations to note, but also to provide the content for them to notes. The later generations of traditional note are mainly from the two plates of the text annotation.

By referring to the later note, you can found that the descendants are annotated based on Guo Pu's annotation. If they agree with Guo Pu's footnotes, most of them are written directly in the back of the text; if dissatisfied or supplement, they would wrote their own viewpoint behind Guo Pu's annotation, and in the form of. Account "somebody thought". Guo Pu's annotation is relatively simple, which provide the direction for future generations, they are mostly use a positive, negative or complementary form, through the wide levy cited further improve, correct the notes of Guo Pu. In addition, there are some content Guo Pu in the form of "unknown" to the content of the notes for future generations to leave enough space to play.

In the After-teeming of Shanhai Jing, Yang Shen is followed by Guo Pu pioneered the style in the direction of attention and commenn. The word "he is good at courtly and gentle, has the rare gifts and bold strategy, be good at research work; whether the class work, the books of all classes of authors or the unofficial novels, are all the scope what he pay close attention" is the pertinent evaluation of Yang Shen. Yang Shen is fond of the ancient Chinese prose, he was the author of < odd word rhyme words $>$ and $<$ ancient Pian works $>$ the two books. Because he has a profound academic foundation in the ancient rhyme pronunciation, that exegesis is an important focus of his Additional Note of Shanhai Jing. In the notes, he often can use his rich knowledge of the place where Guo Pu did not comment notes. Such as the sentence of "Where the beast are mostly of david's deer zhi” in Baoshan of the twelfth of Zhongci Jing, and Here the "deer zhi" is not commented by Guo Pu, while Yangshen sited it as muntjac. Such comments made up the lack of Guo Pu's notes, and also been recognized by the scholars of the future generations, Yuan Ke used his statement. In addition, to the place which was not clear in Guo Pu notes, Yang Shen conducted a further supplement.

\subsection{Annotation method: Use their personal seen knowledge to prove the truth of Shanhai Jing.} GuoPu's understanding of Shanhai Jing's text has an important guiding significance for its annotation method. In order to prove the authenticity of Shanhai Jing's content, Guo Pu opened the first note textual style. In order to prove the authenticity of Shanhai Jing, Guo Pu mainly uses two types of comments. On the one hand, he draw the history and reality objects which were similar with the things signed in Shanhai Jing into his notes; which includes both the historical objects and the involves cultural relics unearthed. On the other hand, he not only cited the records of the related images in the ancient books, he also cited the reality of similar rumors such as the person, the time, and the place. In short, through cited the kind of different forms of information, GuoPu proved that the concept of its ideas to provide convincing intuitive, three-dimensional all kinds of evidence.

In addition to the traditional method of using quoted literature, Guo Pu focused on the use of authentic materials and the unearthed cultural relics as the way of illustration. Compared to literature, 
this kind of evidence is more convincing. The Ming Dynasty scholar Yang Shen well inherited GuoPu's annotation method.

Yangshen has the experience of exiled in Yunnan for 38 years, during this period he greatly enriched his knowledge. In his Additional Note of Shanhai Jing, he always the things what he saw when his exile in Yunnan as the evidences, so as to reinforce the authenticity of the Shanhai Jing's content and correct the uncertainty of Guopu's comments. For example, to the lection signed in South of Dahuang Jing as "There is a place where people have three bodies. And the emperor Jun's wife Ehuang was borned in this country where the people with three bodies, her name is Yao, she like to eat the broomcorn millet, her attendants are four birds." Guopu's commented as "Should be her future generations", while Yangshen commented as "How can the barbarians in remote places have a family name. Which maybe the name of the land rewarded by Xiayu, this is the sage's Enze all over the Quartet's sake. This is what said "through sound education square" in the book of Shangshu. Now there is only one family name in some remote parts of the south of the country, which is the historical inheritance.” Seen from the content, Guo Pu's notes are just a rough deduction, and Yang Shen's notes are much more detailed. Yang Shen in the annotation process using both ancient literature and the use of their own in Yunnan saw ethnographic materials. Therefore, his conclusion is more convincing than Guo Pu's. At the same time, Yang Shen also used his sense of the Yunnan Bai Yi to customs properly for Shanhai Jing related text annotation, to supplement Guopu's notes. Such as when noted the "Dog seal country" signed in North of Hainei Jing, to the sentence "there is a woman kneeling to eat the food in the cup", for the women's movement in the end trying to do, Guopu noted as" She was with food and wine ". This means that there was a woman kneeling there in with food and wine. While Yangshen according to his own folk material, and further on the woman why there will be such a move to make a detailed description, He said: Today, Yunnan is a place where there have a lot of ethnic groups, and where there are many beautiful women. There's a man irrespective of caste, each have several wives. The wives there are very respectful of their husband, as is to treat the monarchy as respect, and they didn't jealous of each other. When the husband and concubine go to bed, the wife is going to serve them, because her husband's position is very important. In her husband eating, changing the time, the wife must kneel to serve, can's look up. Recently, in order to carry out the training of ginger dream, I personally to this place. After coming back, I jokingly said to others: "Chinese said Zhou Wenwang empresses are not jealous. In the place of Baiyi, the women there were very respectful to their husbands. Kneeling to wait on her husband to eat, is probably the custom there." In this text notes, Yangshen was the special information he had witnessed the marriage customs of ethnic minorities in Yunnan to verify the rationality of Shanhai Jing. Compared with Guo Pu's conjecture, Yang Shen's interpretation of this cross-cultural perspective more credibility and persuasion, can also confirm Shanhai Jing authentic content.

\subsection{The spirit of seeking truth and being pragmatic.}

In his Shanhai Jing Note, Guo Pu followed the "seeking truth from facts" principle, so-called "To know, admit what you don't know." His such attitude and Chuang-tzu's "people know if they don't know" cognitive position are consistent. It is recognizing the limitations of human understanding, so Guopu use an open view in seeing the world. In the specific comments, if Guo Pu can collect all kinds of information, he will use a simple image directly in the annotation language will be reflected. On the contrary, he would be use "not clear" "not heard" and other concise forms to note. This pragmatic spirit has a profound influence on the later scholars.

Affected by the spirit of Guopu's such commentary, the later scholars much like to make their own arguments more informative by using the extensive cited notes. Yangshen's Additional Note of Shanhai Jing is the source of this. ${ }^{[7]}$ Based on the annotation by Guo Pu, In the process of annotation, Yang Shen fully used his wealth of knowledge and the investigation of the valuable information. On the one hand, he would speak out about the mistakes of Guopu's comments; on the other hand, He would be enrich and improve in the places where Guopu's comments are not comprehensive enough.

To the Mountain of the pineal gland signed in the West Mountain Jing, the original is "The name is Tong canal, its body is like a pheasant, with black body and barefoot.” To the "Tong”, Guopu noted 
as "a bird named Tong"; while Yangshen noted as "tong canal is Yong canal, and the South central Tonghai County has the bird, the old phonetic as Tong is wrong.” Yangshen's note is not only more detailed, also bluntly pointed out here annotation errors of GuoPu. Although Yangshen is highly praised for Guopu, but the scholar's style of seeking truth and pragmatism made him more respect for the fact, and be brave enough to correct the mistakes or shortcomings of Guo Pu. It is also because of this that Shanhai Jing was able to spread so far.

\section{Summary}

As the first famous scholar noted Shanhai Jing, Guopu and his Shanhai Jing Note played an important role in the process of spreading. Which not only provide a variety of reference for the later scholars, but also provide a platform for future scholars to note. Yangshen extensive knowledge and rich horizons provide adequate resources for him to adding Guopu's Shanhai Jing note. In order to achieve the purpose of glory by writing, Yangshen turned his attention to Shanhai Jing; under the influence of Guopu, he noted Shanhai Jing and made some aspects rich and perfect in the book. ${ }^{[8]}$

\section{Acknowledgments}

This paper was funded by the project of Social Science Foundation of Jilin Province, which project numbered 2016BS48.

$\mathrm{Yao} \mathrm{Xu}$ is the Corresponding author of this paper.

\section{References}

[1] [Tang] X.L. Fang, Book of Jin, 6 Copies, Zhong Hua Press, Beijing, 1986. pp. 1902.

[2] S.Y. Yi, Study on GuoPu's Shanhai Jing Note. Ph.D. Northeast Normal University of Chinese literature, Changchun, 2013. pp.3-8.

[3] ZH. Yang. Research the summary on Yangshen in the past thirty years, Journal of Chongqing University of Arts and Sciences, Vol. 29 No.2, pp.131-137.

[4] Z.Q. Luo, From Yang Shen's view of literature to see the phenomenon of the development of literary thought, Journal of Capital Normal University (Social Sciences Edition), 2009 No.4, pp.90-100.

[5] X.J. Cong, Research of Shanhai Jing Summarized Book. Journal of Xingtai Vocational and Technical College, Vol. 31, (2001) No.3 pp. 42-46.

[6] F. sun, Current situation and Prospect of Yang Shen research, Journal of Southwest University of Science and Technology (Philosophy and Social Science Edition), Vol. 28, (2011) No.2 pp. 78-84.

[7] R.Q. Jin, The two thousand years of research review of Shanhai Jing, Journal of Xinyang Normal University (Philosophy and Social Sciences Edition), Vol. 20, (2000) No.4 pp. 42-46.

[8] B.T. Zhang, Research on ancient editions of Shanhai Jing-also on partial Shanhai Jing with annotations and commentaries, Journal of Fuqing Branch of Fujian Normal University. 2013 No.3, pp.1-4. 